\title{
Finite element shear models of thin-walled structures of water management systems of agro-industrial complex
}

\author{
Yuriy Klochkov ${ }^{1, *}$, Tlek Ishchanov ${ }^{1}$, Alexandr Andreev ${ }^{1}$, and Mikhail Klochkov² \\ ${ }^{1}$ Volgograd State Agrarian University, 400002 Volgograd, Russia \\ ${ }^{2}$ Moscow State University, 119992 Moscow, Russia
}

\begin{abstract}
The article presents an algorithm for constructing a finite element model of deformation of thinwalled structures such as pipelines, tanks, bunkers included in the structure of the agro-industrial complex. The proposed model takes into account the deformation of the transverse shear. As a finite element, it is proposed to use a quadrangular fragment of the middle surface of a thin-walled structure of the agroindustrial complex with nodes located at its vertices. The components of the displacement vector and their partial derivatives of the first order with respect to curvilinear coordinates, as well as the components of the normal rotation angle vector, were chosen as the required unknowns. In the construction of the finite element model, the developed interpolation procedure was used for the components of the displacement vector and the component of the normal rotation angle vector as components of vector fields. The efficiency of the proposed finite element models in terms of a significant increase in the accuracy of calculations, the convergence of the computational process and the adequacy of the results to the physical meaning of the problem was proved on the numerical example of the calculation of the pipeline fragment.
\end{abstract}

\section{Introduction}

The development of the agro-industrial complex of Russia requires the introduction of modern computing digital technologies in engineering and calculation practice of designing structures of the agro-industrial complex, many of which belong to the class of thinwalled. The examples of such structures include irrigation, drainage, watering pipelines, tanks, bunkers for various purposes and much more.

The search for optimal shapes and sizes of such thinwalled structures leads to the need to simulate the deformation processes of the latter based on the use of finite element shear models for the analysis of their stress-strain state (SSS).

In most common currently, computer complexes, such as Ansys and the like, use simplified finite element models constructed on the basis of the standard FEM interpolation procedure, a separate component of the displacement vector components as scalar fields [1-25]. The above interpolation procedure allows obtaining correct solutions only when using a Cartesian coordinate system.

At the same time, when calculating thin-walled structures of water management systems, which are shells or their fragments, it is convenient to use curvilinear coordinate systems, which introduce additional problems into computational algorithms, such as the problem of accounting for displacements of a finite element as an absolutely rigid body. The solution of this problem is possible by using the interpolation procedure components of the displacement vector as components of vector fields.

The article describes the main stages of construction of finite element models of shear of thin-walled constructions calculation of AIC based on the use of quadrilateral element discretization, the stiffness matrix which is based on invariant interpolation procedure component of the displacement vector and the component vector of angles of rotation of normals as components of vector fields.

\section{Materials and methods}

\subsection{Geometric relations}

When obtaining the dependencies between the components of the strain tensor and the components of the displacement vector in the calculation of thin-walled structures of water systems, the hypothesis of a straight normal [26] is usually used, according to which the displacement vector of the point $\mathrm{M}^{0 \zeta}$, spaced from the median surface by some distance $\zeta$, is determined by the formula

$$
\mathbf{v}=\mathbf{v}+\zeta\left(\mathbf{a}-\mathbf{a}^{0}\right),
$$

where $\mathbf{a}$ and $\mathbf{a}^{0}$ are orts of normals to the median surface in the deformed and initial States.

Included in (1) the displacement vector of the point $\mathrm{M}^{0}$ of the median surface can be represented by the components of the local basis of this point

\footnotetext{
* Corresponding author: Klotchkov@bk.ru
} 


$$
\mathbf{v}=v^{1} \mathbf{a}_{1}^{0}+v^{2} \mathbf{a}_{2}^{0}+v \mathbf{a}^{0} .
$$

The use of (1) entails the appearance in the Cauchy relations of the second derivatives of the normal component of the displacement vector [26, 27]. In addition, in such relations there are no deformations that take into account the transverse shear, which is necessary in the calculations of short-span structures, as well as structures with rigid support, sliding and other types of seals that prohibit the angle of rotation. Therefore, the most appropriate is to write the displacement vector $\mathbf{V}$ of the point $\mathrm{M}^{0 \zeta}$, spaced from the median surface at a distance $\zeta$, in the following formulation [28]

$$
\mathbf{V}=\mathbf{v}+\zeta\left(\boldsymbol{\gamma}_{\Omega} \times \mathbf{a}^{0}\right)
$$

where $\boldsymbol{\gamma}_{\Omega}=-\gamma^{2} \mathbf{a}_{1}^{0}+\gamma^{1} \mathbf{a}_{2}^{0}+\Omega \mathbf{a}^{0}$ is a vector of angles of rotation normal [3]

After executing the vector product, the formula (3) takes the form

$$
\mathbf{v}=\mathbf{v}+\zeta \boldsymbol{\gamma}
$$

where $\boldsymbol{\gamma}=\gamma^{1} \mathbf{a}_{1}^{0}+\gamma^{2} \mathbf{a}_{2}^{0}$.

The position of the point of the thin-walled structure before and after deformation is determined by the radius vectors

$$
\mathbf{R}^{0 \zeta}=\mathbf{R}^{0}+\zeta \mathbf{a}^{0} ; \mathbf{R}^{\zeta}=\mathbf{R}^{0 \zeta}+\mathbf{V},
$$

where $\mathbf{R}^{0}$ is the radius vector of the point of the median surface of the thin-walled structure.

Differentiation (5) on global coordinates $\alpha, \beta$ vectors of bases of any point of a thin-walled construction are defined

$$
\mathbf{g}_{\alpha}^{0}=\mathbf{R}_{, \alpha}^{0 \zeta} ; \mathbf{g}_{\alpha}=\mathbf{R}_{, \alpha}^{\zeta},
$$

By scalar products (6) covariant components of the metric tensor can be obtained before and after deformation of the thin-walled structure

$$
g_{\alpha \beta}^{0}=\mathbf{g}_{\alpha}^{0} \cdot \mathbf{g}_{\beta}^{0} ; g_{\alpha \beta}=\mathbf{g}_{\alpha} \cdot \mathbf{g}_{\beta},
$$

Deformations at an arbitrary point of a thin-walled structure can be calculated by the continuum mechanics ratio [29]

$$
\varepsilon_{\alpha \beta}^{\zeta}=0,5\left(g_{\alpha \beta}-g_{\alpha \beta}^{0}\right)
$$

Here and below, the subscripts $\alpha$ and $\beta$ consistently take the values $1,2,3$. And 1 and 2 correspond to the surface curvilinear coordinates, and the figure 3-the coordinate in the direction perpendicular to the surface.

\subsection{Interpolation procedure in a quadrangular finite element.}

The thin-walled construction is modeled by a set of quadrangular fragments of the middle surface with nodes $1, \mathrm{~m}, \mathrm{n}, \mathrm{p}$. The components of the displacement vector (2) and their partial derivatives with respect to the curvilinear coordinates of the surface $\alpha$ and $\beta$, as well as the components of the rotation angle vector of the normal $\gamma$ (4) were chosen. Thus the column of nodal unknowns in the global curvilinear coordinate system has the following form

$$
\left\{U_{y}\right\}^{T}=\left\{\left\{v_{y}^{1}\right\}^{T}\left\{v_{y}^{2}\right\}^{T}\left\{v_{y}\right\}^{T}\left\{\gamma^{1}\right\}^{T}\left\{\gamma^{2}\right\}^{T}\right\},
$$

where

$$
\begin{array}{r}
\left\{w^{l} w^{m} w^{n} w^{p} w_{, \alpha}^{l} w_{, \alpha}^{m} w_{, \alpha}^{n} w_{, \alpha}^{p} w_{, \beta}^{l} w_{, \beta}^{m} w_{, \beta}^{n} w_{, \beta}^{p}\right\} ; \\
\left\{\gamma^{\rho}\right\}^{T}=\left\{\gamma^{l} \gamma^{m} \gamma^{n} \gamma^{p}\right\} ; \rho=1,2 .
\end{array}
$$

Here, w refers to the component of the displacement vector $v^{1}, v^{2}$ or $v$.

When determining the SSS of thin-walled structures with curvilinear surface forms, it is most correct to apply the vector form of the interpolation procedure, according to which the following interpolation dependences for the displacement vector and the normal rotation angle vector are used

$$
\left.\mathbf{v}=\underset{1 \times 12}{\{\psi\}^{T}[M]} \underset{12 \times 12}{\left\{\mathbf{v}_{y}\right\}}\right\} ; \gamma=\underset{1 \times 4}{\{\chi\}^{T}} \underset{4 \times 1}{\left\{\boldsymbol{\gamma}_{y}\right\}}
$$

where $\left.\{\psi\}^{T} ;\{\chi\}^{T}\right\}^{T}$ - matrices - rows of form functions whose elements are represented by products of Hermite polynomials of the third degree and bilinear functions of local coordinates $-1 \leq \xi, \eta \leq 1$, respectively; [M] transition matrix from nodal unknowns in the local coordinate system to nodal unknowns in the global coordinate system.

The columns of vector nodal unknowns included in the right parts (10) have the following form

$$
\begin{aligned}
& \underset{1 \times 12}{\left\{\mathbf{v}_{y}\right\}^{T}}=\left\{\mathbf{v}^{l} \mathbf{v}^{m} \mathbf{v}^{n} \mathbf{v}^{p} \mathbf{v}_{, \alpha}^{l} \mathbf{v}_{, \alpha}^{m} \mathbf{v}_{, \alpha}^{n} \mathbf{v}_{, \alpha}^{p} \mathbf{v}_{, \beta}^{l} \mathbf{v}_{, \beta}^{m} \mathbf{v}_{, \beta}^{n} \mathbf{v}_{, \beta}^{p}\right\} ;,
\end{aligned}
$$

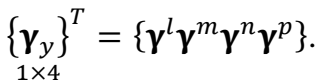

Representing the displacement vectors and the rotation angle vectors of the normal (11) components of the nodal local bases, the relations (10) can be written as

$$
\begin{aligned}
& \mathbf{v}=\underset{1 \times 12}{\{\psi\}^{T}} \underset{12 \times 12}{[M]} \underset{12 \times 36}{\left[\mathbf{A}_{v}\right]}\left\{d_{y}\right\} ; \gamma=\underset{1 \times 4}{\{\chi\}^{T}} \underset{4 \times 8}{\left[\mathbf{A}_{\gamma}\right]}\left\{\gamma_{8 \times 1}\right\}, \\
& \left\{d_{y}\right\}^{T}=\left\{v^{1 l} v^{2 l} v^{l} v^{1 m} v^{2 m} v^{m} v^{1 n} v^{2 n} v^{n} \ldots\right\} ;
\end{aligned}
$$

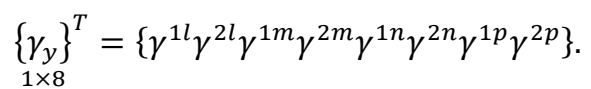

Included in (12) matrices $\left[\mathbf{A}_{v}\right]$ and $\left[\mathbf{A}_{\gamma}\right]$, containing vectors of local bases of finite element nodes, can be represented as matrix sums

$$
\begin{gathered}
{\left[\mathbf{A}_{v}\right]=\mathbf{a}_{1}^{0}\left[A_{v}^{1}\right]+\mathbf{a}_{2}^{0}\left[A_{v}^{2}\right]+\mathbf{a}^{0}\left[A_{v}^{3}\right] ;\left[\mathbf{A}_{\gamma}\right]=} \\
=\mathbf{a}_{1}^{0}\left[A_{\gamma}^{1}\right]+\mathbf{a}_{2}^{0}\left[A_{\gamma}^{2}\right] .
\end{gathered}
$$

Taking into account (13), (2) and (4), the ratios (12) will take the following form

$$
v^{1} \mathbf{a}_{1}^{0}+v^{2} \mathbf{a}_{2}^{0}+v \mathbf{a}^{0}=
$$




$$
\begin{aligned}
& =\{\psi\}^{T}\left(\mathbf{a}_{1}^{0}\left[A_{v}^{1}\right]+\mathbf{a}_{2}^{0}\left[A_{v}^{2}\right]+\mathbf{a}^{0}\left[A_{v}^{3}\right]\right)[G]\left\{d_{y}\right\} \\
& \gamma^{1} \mathbf{a}_{1}^{0}+\gamma^{2} \mathbf{a}_{2}^{0}=\{\chi\}^{T}\left(\mathbf{a}_{1}^{0}\left[A_{\gamma}^{1}\right]+\mathbf{a}_{2}^{0}\left[A_{\gamma}^{2}\right]\right)\left\{\gamma_{y}\right\} .
\end{aligned}
$$

From (14) it is possible to obtain interpolation expressions for the displacement vector component and the normal rotation angle vector component when implementing the vector form of the interpolation procedure

$$
\begin{gathered}
v^{1}=\{\psi\}^{T}\left[A_{v}^{1}\right][G]\left\{d_{y}\right\} ; v^{2}=\{\psi\}^{T}\left[A_{v}^{2}\right][G]\left\{d_{y}\right\} \\
v=\{\psi\}^{T}\left[A_{v}^{3}\right][G]\left\{d_{y}\right\} ; \gamma^{1}=\{\chi\}^{T}\left[A_{\gamma}^{1}\right]\left\{\gamma_{y}\right\} \\
\gamma^{2}=\{\chi\}^{T}\left[A_{\gamma}^{2}\right]\left\{\gamma_{y}\right\}
\end{gathered}
$$

Further formation of the stiffness matrix and the external load column of the finite element is carried out by minimizing the Lagrange functional in a standard FEM manner [1-25].

\section{Calculation example}

An elliptical cylinder loaded in the middle with a concentrated force $\mathrm{P}=453.6 \mathrm{~N}$ and having a hinged support on the diametrically opposite side preventing vertical displacement was calculated (Fig.1). At the ends of the cylinder has a sliding seal. The initial data had the following values: cylinder length $\mathrm{L}=26.29 \cdot 10^{-2} \mathrm{~m}$; shell thickness $\mathrm{h}=0.24 \cdot 10^{-2} \mathrm{~m}$; elastic modulus $\mathrm{E}=0.738 \cdot 10^{5}$ $\mathrm{MPa}$; Poisson's ratio $v=0.3125$. Due to the presence of symmetry planes, the $1 / 4$ part of the shell was calculated.

The calculations were performed according to two variants. In the first variant, the standard interpolation procedure of the components of the displacement vector and the rotation angle vector of the normal as scalar values was used for the arrangement of the stiffness matrix and the nodal forces column of the quadrangular FE [1-25]. In the second variant, interpolation of the components of the displacement vector and the normal rotation angle vector as components of the vector fields (10) - (15) was implemented.

Was initially designed in a circular cylinder, i.e. the parameters of the ellipse which is the cross section of the cylinder was taken equal to $b=c=12.58 \cdot 10^{-2} \mathrm{~m}$. the calculation Results for two variants of the interpolation procedure are presented in table № 1, which shows the variation of the deflection under concentrated force (point A) and the value of the normal $\sigma^{11}, \sigma^{22}$ and $\sigma^{13}$ shear, stresses at points 1 and 2 at the end of the shell to the inner $\sigma_{\text {int }}$, and $\sigma_{\text {out }}$ of external surfaces of the shell depending on the density of the mesh discretization of shell.

The analysis of the data presented in Table 1 shows that in both variants of calculation there is a satisfactory convergence of the computational process, and the variation values of deflection and stress are almost the same or close enough to each other for the same sampling grids. In addition, it should be noted the practical coincidence of the stress values at points 1 and 2 in both versions of the calculation, which is due to the symmetry of the chosen design scheme of the shell.
Further, the calculation of the thin-walled structure of the AIC in the form of an elliptical cylinder was performed at the ratio of the cross-sectional parameters $\mathrm{b} / \mathrm{c}=4 / 1$. All other original data retained the same values.

The results of the calculation of the elliptical cylinder are presented in Table 2, the structure of which coincides with Table 1. An analysis of the data in Table 2 shows that there are very significant differences between the two calculation options. Thus, in the variant of scalar interpolation procedure there is a slow rate of convergence of the computational process, which can not be considered satisfactory.

The values of deflection under the concentrated force, as well as the stress values were significantly lower compared to the option of using the vector method of interpolation procedure. It should also be noted a significant difference between the stress values at points 1 and 2 of the cylinder in the first version of the calculation.

In the second variant of calculation it is possible to observe steady convergence of computing process. In addition, it should be noted that the stress values at points 1 and 2 of the cylinder are exactly the same, regardless of the sampling grid used.

Symmetry of the computational scheme of the elliptic shell (Fig.1) also assumes the equality of normal stresses at the point $\mathrm{A}$ of the application of the concentrated force $\mathrm{P}$ and at the point $\mathrm{B}$ of the hinge support. Figures 2 and 3 show graphs of changes in the normal stress ratios

$$
w_{\text {int }}^{1}=\left(\sigma_{\text {int }}^{11}\right)_{A} /\left(\sigma_{\text {int }}^{11}\right)_{B} \text { and } w_{\text {out }}^{2}=\left(\sigma_{\text {out }}^{22}\right)_{A} /\left(\sigma_{\text {out }}^{22}\right)_{B}
$$
at points $\mathrm{A}$ and $\mathrm{B}$, depending on the ratio of the semiaxes of the ellipse b/a, which is the cross-section of the cylinder, with a scalar (Fig. 2) and vector (Fig. 3) variants of the interpolation procedure.

The sampling grid $21 \times 21$ was used in the construction of these graphs. As can be seen from figure 2 , when using the scalar interpolation procedure, $w_{i n t}^{1}$ and $w_{\text {out }}^{2}$ are equal to 1 only when the $v / s$ ratio is one and two. With increasing the ratio $v / s$, with two to four values $w_{\text {int }}^{1}$ and $w_{\text {out }}^{2}$ dramatically increase to unacceptable values. Using a vector interpolation procedure $w_{\text {int }}^{1}$ and $w_{\text {out }}^{2}$ remains equal to 1 at any ratio $v / s$ (Fig. 3)

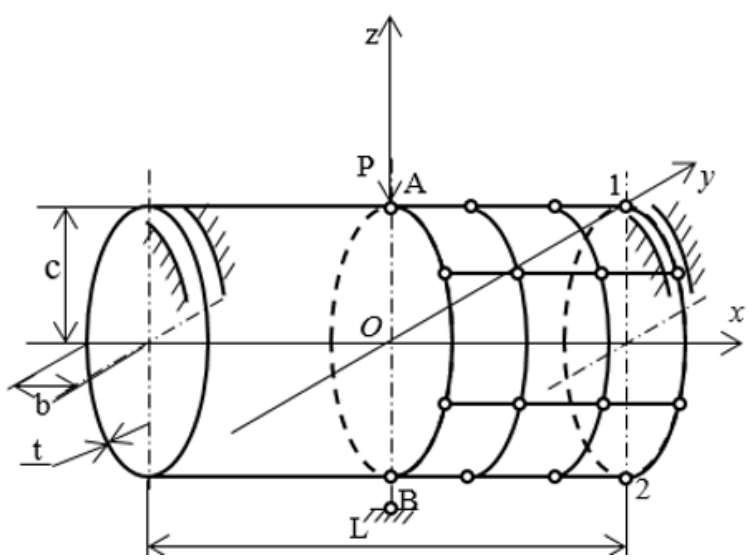

Fig. 1. Design scheme of the pipeline fragment. 
Table 1. Stresses in characteristic sections of a circular cylinder

\begin{tabular}{|c|c|c|c|c|c|c|c|}
\hline \multirow{4}{*}{$\begin{array}{l}\text { Coordinates of } \\
\text { points: } x \\
\left(10^{-2} \mathrm{~m}\right), \mathrm{t}(\mathrm{rad})\end{array}$} & \multirow{4}{*}{$\begin{array}{l}\text { SSS parameters: } \\
\mathrm{v}\left(10^{-2} \mathrm{~m}\right), \sigma \\
(\mathrm{MPA})\end{array}$} & \multicolumn{6}{|c|}{ Variant of interpolation procedure } \\
\hline & & \multicolumn{3}{|c|}{ Conventional interpolation } & \multicolumn{3}{|c|}{ Vector form of the interpolation } \\
\hline & & \multicolumn{6}{|c|}{ Sampling grid } \\
\hline & & $9 \times 9$ & $17 \times 17$ & $21 \times 21$ & $9 \times 9$ & $17 \times 17$ & $21 \times 21$ \\
\hline $\begin{array}{c}\text { p. A, } \\
x=0.0, \\
t=0.0\end{array}$ & $\mathrm{v}$ & 0.5168 & 0.5547 & 0.5608 & 0.5067 & 0.5574 & 0.5627 \\
\hline \multirow{6}{*}{$\begin{array}{c}\text { p. } 1, \\
x=L / 2, \\
t=0.0\end{array}$} & $\sigma_{\text {int }}^{11}$ & 14.78 & 13.80 & 13.15 & 13.40 & 13.59 & 13.02 \\
\hline & $\sigma_{\text {out }}^{11}$ & -13.20 & -13.05 & -12.47 & -12.17 & -12.85 & -12.34 \\
\hline & $\sigma_{\text {int }}^{22}$ & 50.42 & 53.41 & 52.86 & 45.92 & 52.77 & 52.46 \\
\hline & $\sigma_{\text {out }}^{22}$ & -46.57 & -54.81 & -55.12 & -43.11 & -54.22 & -54.74 \\
\hline & $\sigma_{i n t}^{13}$ & -3.26 & -5.68 & -6.67 & -3.04 & -5.60 & -6.61 \\
\hline & $\sigma_{\text {out }}^{13}$ & -3.26 & -5.68 & -6.67 & -3.04 & -5.60 & -6.61 \\
\hline \multirow{6}{*}{$\begin{array}{c}\text { p. } 2, \\
x=L / 2, \\
t=\pi\end{array}$} & $\sigma_{\text {int }}^{11}$ & 14.54 & 13.79 & 13.15 & 13.40 & 13.59 & 13.02 \\
\hline & $\sigma_{\text {out }}^{11}$ & -13.43 & -13.06 & -12.50 & -12.17 & -12.85 & -12.34 \\
\hline & $\sigma_{\text {int }}^{22}$ & 49.68 & 53.36 & 52.84 & 45.92 & 52.77 & 52.46 \\
\hline & $\sigma_{\text {out }}^{22}$ & -47.25 & -54.86 & -55.14 & -43.11 & -54.22 & -54.74 \\
\hline & $\sigma_{\text {int }}^{13}$ & -3.26 & -5.68 & -6.67 & -3.04 & -5.60 & -6.61 \\
\hline & $\sigma_{0 \nu t}^{13}$ & -3.26 & -5.68 & -6.67 & -3.04 & -5.60 & -6.61 \\
\hline
\end{tabular}

Table 2. Stresses in characteristic sections of an elliptical cylinder

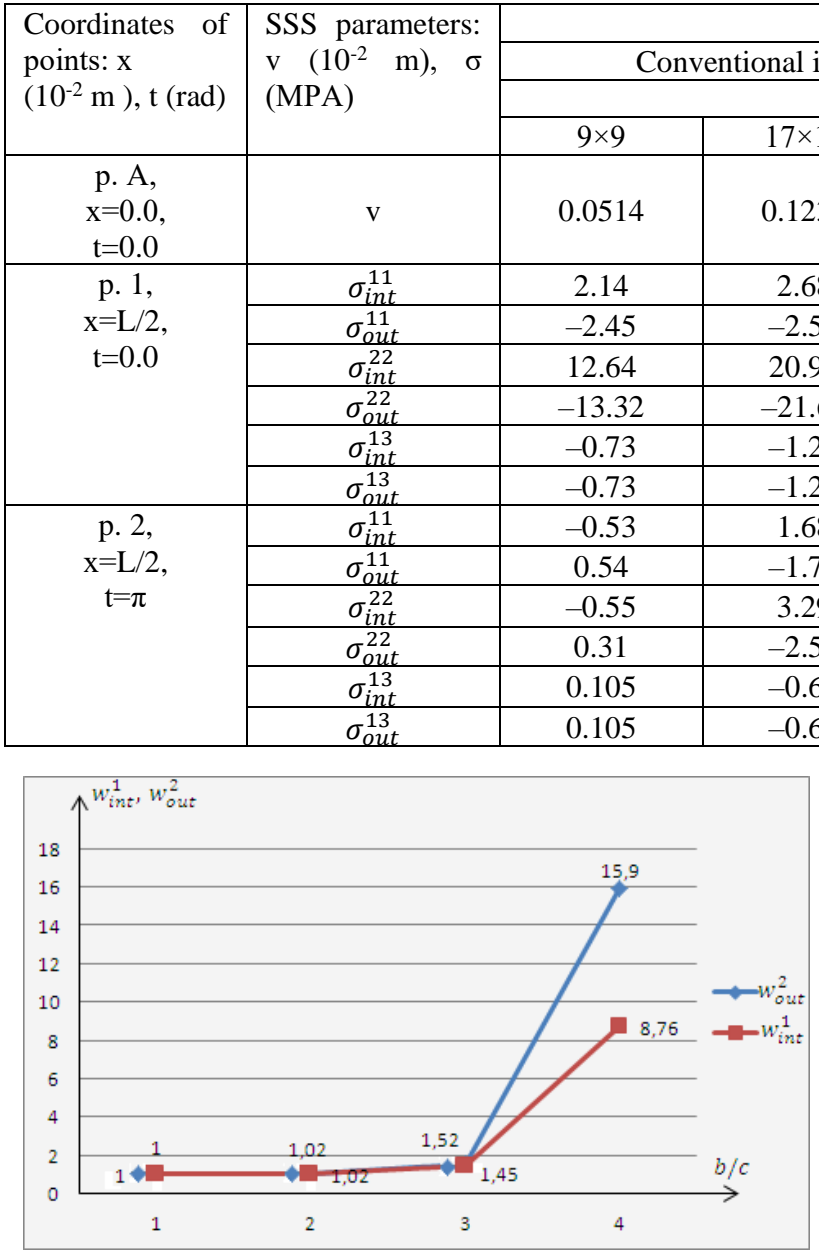

Fig. 2. Comparative diagram in the conventional interpolation procedure
Variant of interpolation procedure

\begin{tabular}{|l|l} 
al interpolation & Vector form of the interpolation
\end{tabular}

\begin{tabular}{|c|c|c|c|c|}
\hline \multicolumn{5}{|c|}{ Sampling grid } \\
\hline $7 \times 17$ & $21 \times 21$ & $9 \times 9$ & $17 \times 17$ & $21 \times 21$ \\
\hline & & & & \\
& 0.1831 & 0.3479 & 0.3925 & 0.3982 \\
\hline 2.68 & & & & \\
\hline-2.57 & -3.57 & 9.23 & 8.14 & 7.72 \\
\hline 20.93 & 24.54 & -8.44 & -7.88 & -7.52 \\
\hline-21.65 & -25.09 & -32.79 & -37.72 & -37.47 \\
\hline-1.27 & -1.94 & -2.20 & -3.52 & -4.07 \\
\hline-1.27 & -1.94 & -2.20 & -3.52 & -4.07 \\
\hline 1.68 & 3.14 & 9.23 & 8.14 & 7.72 \\
\hline-1.71 & -3.14 & -8.44 & -7.88 & -7.52 \\
\hline 3.29 & 10.43 & 37.84 & 39.06 & 38.30 \\
\hline-2.52 & -9.53 & -32.79 & -37.72 & -37.47 \\
\hline-0.68 & -1.63 & -2.20 & -3.52 & -4.07 \\
\hline 0.68 & -1.63 & -2.20 & -3.52 & -4.07 \\
\hline
\end{tabular}

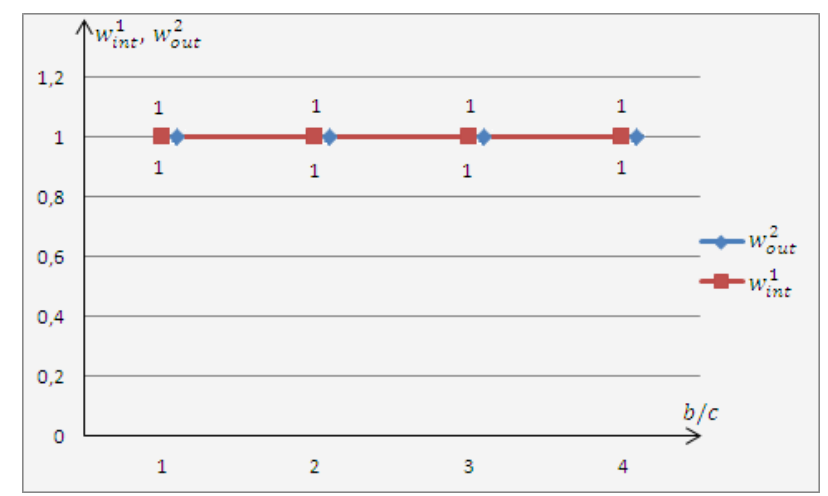

Fig. 3. Comparative chart when the vector forms the interpolation 


\section{Conclusion}

The following conclusions can be drawn from the analysis of tabular data.

1. The use of the standard scalar interpolation procedure [1-25] allowed obtaining the correct VAT parameters only when calculating the circular cylinder, i.e. the simplest version of the thin shell.

2. When calculating thin-walled constructions agriculture, non-circular cylinders (for example, elliptical cylinders), to obtain the correct values of VAT needed to apply the vector version of the interpolation procedure because the interpolation component of the displacement vector and the component vector of the rotation angle of the normal components of vector fields allows considering not only changes in the aforementioned component, but also a change of the local basis vectors point in the middle surface of a thin shell in the process of its deformation when using curvilinear coordinate systems.

\section{Acknowledgments}

The study was supported by the Russian Foundation for Basic Research and the Volgograd Region Administration as part of research project No. 19-41343003 r_mol_a.

\section{References}

1. K.-J. Bathe, F. Brezzi, L.D. Marini, The MITC9 shell element in plate bending: mathematical analysis of a simplified case Comput. Mech. 47(6), 617-626 (2011)

2. A.I. Golovanov, O.N. Tyuleneva, A.F. Shigabutdinov, Finite element Method in the statics and dynamics of thin-walled structures (Fizmatlit, Moscow, 2006)

3. R.A. Kayumov, I.Z. Muhamedova, F.R. Shakirzjanov, B.F. Tazyukov, Parameter determination of hereditary models of deformation of composite materials based on identification method J. of Phys.: Conf. Ser. Current Probl. 012006 (2018)

4. O.C. Zienkiewicz, R.L. Taylor, The Finite Element Method for Solid and Structural Mechanics (Elsevier, Oxford, 2005)

5. V.K. Belov, L.P. Zheleznov, T.S. Ognyanova, Nonlinear deformation and stability of a noncircular cylindrical shell under combined loading with bending and twisting moments J. of Applied Mech. and Techn. Phys. 59(2), 352-358 (2018)

6. Nguyen Nhung, Waas Anthonym, Nonlinear, finite deformation, finite element analysise ZAMP. Z. Angew. math. and Phys. 67(9), 35/1-35/24 (2016)

7. S.L. Paznanova, G.P. Vasilev, P.S. Dineva, G.D. Manolis, Dynamic analysis of nanoheterogeneities in a finite-sized solid by boundary and finite element methods Int. J. Solids and Struct. 80, 1-18 (2016)

8. P. Wriggers, B. Reddy, W. Rust, B. Hudobivnik, Efficient virtual element formulations for compressible and incompressible finite deformations Comput. Mech. 60, 253-268 (2017)

9. A.S. Solodovnikov, S.V. Sheshenin, Numerical study of strength properties for a composite material with short reinforcing fibers Moscow Univer. Mech. Bull. 72(4), 94-100 (2017)

10. V.N. Paimushin, S.A. Kholmogorov, M.V. Makarov, D.V. Tarlakovskii, A. Lukaszewicz, Mechanics of fiber composites: forms of loss of stability and fracture of test specimens resulting from three-point bending tests ZAMM. Zeitschrift fur Angewandte Mathem. und Mech. 99(1), e201800063 (2019)

11. L.U. Sultanov, Analysis of finite elasto-plastic strains. Medium kinematics and constitutive equations Lobachevskii J. of Mathem. 37(6), 787-793 (2016)

12. A.I. Abdrakhmanova, L.U. Sultanov, Numerical modelling of deformation of hyperelastic incompressible solids Mat. Phys. and Mech. 26(1), 30-32 (2016)

13. L.P. Zheleznov, V.V. Kabanov, D.V. Boiko, Nonlinear deformation and stability of discrete reinforced elliptical cylindrical composite shells under torsion and internal pressure Russian Aeronautics 61(2), 175-182 (2018)

14. I.V. Stankevich, S.S. Volkov, Algorithms for solving MDTT boundary value problems taking into account creep deformation Mathematics and mathematical modeling 1, 1-14 (2018)

15. A.M. Belostotsky, P.A. Akimov, I.N. Afanasyeva, T.B. Kaytukov, Contemporary problems of numerical modelling of unique structures and buildings Int. J. for Computational Civil and Structural Engin. 13(2), 9 (2018)

16. A.M. Belostotsky, A.L. Potapenko, P.A. Akimov, Universal software system "Stadyo" for the numerical solution of linear and nonlinear problems of the field theory, statics, stability and dynamics of spatial combined systems: general parameters and superelemental features Int. J. for Computational Civil and Structural Engin. 14(3), 26-41 (2018)

17. V.N. Paimushin, I.B. Badriev, S.A. Kholmogorov, Consistent equations of nonlinear multilayer shells theory in the quadratic approximation Lobachevskii J. of Mathem. 40(3), 349-363 (2019)

18. V.V. Lalin, E.V. Zdanchuk, Conditions on the surface of discontinuity for the reduced cosserat continuum Mat. Phys. and Mech. 31(1-2), 28-31 (2017)

19. A.V. Ignatiev, V.A. Ignatiev, E.A. Gamzatov, Calculation of thin plates by the finite element method in the form of the classical mixed method with the exclusion of displacements of finite 
elements as a rigid whole in Proc. of higher educational institutions. Construction 3(711), 5-13 (2018)

20. V.V. Galishnikova, P. Ja. Pahl, Analysis of frame buckling without sidesway classification Structural mech. of engin. Struct. and struct. 14(4), 299-312 (2018)

21. K.A. Skoptsov, S.V. Sheshenin, V.V. Galatenko, A.P. Malakho, O.N. Shornikova, V.V. Avdeev, V.A. Sadovnichy, Particle simulation for predicting effective properties of short fiber reinforced composites Int. J. of Applied Mech. 8(2), 1650016 (2016)

22. L. Stupishin, A. Kolesnikov, K. Nikitin, Variable form forming investigation for flexible shallow shells on circular base Asian J. of Civil Engin. 18(2), 163-171 (2017)

23. Yu.Ya. Tyukalov, Finite element models in stresses for plane elasticity problems Civil Engin. J. 1(77), 23-37 (2018)
24. G.A. Genius, K.P. Pyatikrestovsky, Issues of long durability of precious reinforced concrete shells Struct. Mech. and calculation of struct. 1(270), 14-19 (2017)

25. V.G. Bazhenov, M.N. Zhestkov, Applicability of a structurally orthotropic model in problems on tension, bending, and stability of densely perforated plates and shells Mech. of Compos. Mat. 53(2), 159-164 (2017)

26. V.V. Novozhilov, Theory of thin shells 380 (St. Petersburg university, St. Petersburg, 2010)

27. R.B. Rickards, Finite element Method in shell and plate theory 248 (Zinatne, Riga, 1988)

28. K.F. Chernykh, Linear theory of shells, 1, 374 (LSU publ. House, Leningrad, 1962)

29. L.I. Sedov, Continuum Mechanics, 1, 536 (Nauka, Moscow, 1976) 